EPJ Web of Conferences 71, 00030 (2014)

DOI: 10.1051/epjconf/20147100030

(C) Owned by the authors, published by EDP Sciences, 2014

\title{
Top quark physics with the CMS experiment
}

\author{
Javier Cuevas ${ }^{1, a}$ \\ ${ }^{1}$ Universidad de Oviedo, Spain
}

\begin{abstract}
An overview of recent top quark measurements in proton-proton collisions at $\sqrt{s}=7$ and $8 \mathrm{TeV}$ in data collected with the CMS experiment at the LHC, using a data sample collected during the years 2011 and 2012 is presented. Measurements of top quark pair production cross sections in several top quark final states are reported, as well as electroweak production of single top quarks in both $\mathrm{t}$ - and $\mathrm{tW}$-channels. The mass of the top quark is estimated by different methods.
\end{abstract}

\section{Introduction}

The top quark is the heaviest elementary particle known, it decays before fragmenting into hadrons therefore providing a unique possibility to measure the properties of a bare quark. The large value of $m_{\text {top }}$ also makes the Yukawa coupling to the Higgs boson to be close to 1 . For this reason, it is often believed that the top quark can play a special role in the electroweak symmetry breaking mechanism. The top quark is part of many possible beyond the Standard Model (BSM) signatures, where it can be a decay mode of, yet unknown, heavy particles. It is also foreseen that new particles can originate from top decays. Furthermore, top-quark production is an important source of background in many searches for physics beyond the standard model. The Large Hadron Collider (LHC) has operated remarkably well in 2011 and 2012, and CMS [1] has collected a large sample of top-quark events and studies of top-quark production have been conducted in various decay channels as well as searches for deviations from the SM predictions.

\section{Inclusive and differential $t \bar{t}$ cross section measurements}

A precise measurement of the $\bar{t}$ production cross section can be used to test the theory of quantum chromodynamics (QCD) at next-to-next-to-leading-order (NNLO) level. It can be also used in global fits of the parton distribution functions (PDF) at NNLO, and allows for extracting an $\alpha_{s}\left(M_{Z}\right)$. A measurement of the $\overline{\mathrm{t}}$ production cross section at $\sqrt{s}=7 \mathrm{TeV}$ was performed in events containing $\mathrm{e}^{+} \mathrm{e}^{-}, \mu^{+} \mu^{-}$, or $\mathrm{e}^{ \pm} \mu^{\mp}$ lepton pairs [2], at least two jets, and a large imbalance in transverse momentum. The measurement is performed using two approaches: a profile likelihood-ratio procedure and an event-counting analysis that relies on the presence of at least one b-tagged jet. The results from the individual dilepton channels and from the two analysis methods are consistent with each other, and are also found to be compatible with previous published measurements. For the profile likelihood-ratio method, the cross section for the combined dilepton channels amounts to $\sigma_{\mathrm{tt}}=161.9 \pm 2.5$ (stat. $)_{-5.0}^{+5.1}$ (syst.) \pm 3.6 (lumi.) pb, in agreement with the prediction of the standard model.

\footnotetext{
a e-mail: fjcuevas@uniovi.es
} 

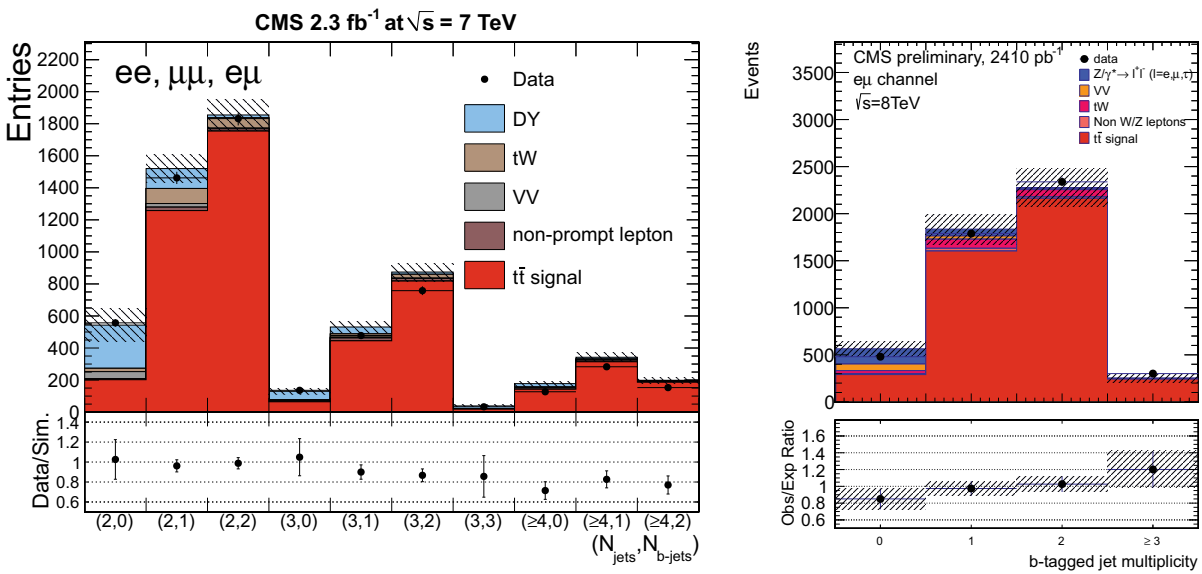

Figure 1. (left) Number of events selected for the three combined channels, as a function of the number of jets and $\mathrm{b}$ jets $\left(\mathrm{N}_{\text {jets }}, \mathrm{N}_{b-\text { jets }}\right)$ in each event. The multiplicity of $\mathrm{b}$ jets in events passing the full event selections (but without cutting in number of $b$ jets), for the emu channel (right) at $8 \mathrm{TeV}$ in dilepton final state.

A similar measurement was performed at proton-proton collisions at $\sqrt{s}=8 \mathrm{TeV}$ in a data sample corresponding to $2.41 \mathrm{fb}^{-1}$ of integrated luminosity collected by the CMS experiment in 2012. The measurement is performed with events with two energetic leptons (electrons or muons) in the final state. Presence of the $b$ jets in the top-quark decays is required. The method used to extract the cross section is a simple and robust counting experiment. The measured cross section is found to be $\sigma_{\mathrm{tt}}=$ $227 \pm 3$ (stat.) \pm 10 (syst.) \pm 10 (lumi.) pb, in agreement with theoretical predictions.

A measurement of the $\bar{t}$ production cross section in the tau dilepton channel [3] $\overline{\mathrm{t}} \rightarrow$ $\left(\ell v_{\ell}\right)\left(\tau_{\mathrm{h}} v_{\tau}\right) \mathrm{b} \overline{\mathrm{b}},(\ell=\mathrm{e}, \mu)$ with data samples corresponding to an integrated luminosity of $2.0-2.2 \mathrm{fb}^{-1}$ collected in proton-proton collisions at $\sqrt{s}=7 \mathrm{TeV}$ is presented. Events are selected by requiring the presence of one electron or muon, two or more jets (at least one jet is b tagged), missing transverse energy, and one hadronically decaying $\tau$ lepton. The largest background contributions come from events where one $\mathrm{W}$ boson is produced in association with jets, and from $\mathrm{t} \overline{\mathrm{t}} \rightarrow \mathrm{W}^{+} \mathrm{bW}-\overline{\mathrm{b}} \rightarrow \ell \nu \mathrm{b} q \overline{\mathrm{q}}^{\prime} \overline{\mathrm{b}}$ events, where one jet is misidentified as the $\tau$, and from $\mathrm{Z} \rightarrow \tau \tau$ events. The measured cross section is $\sigma_{\mathrm{tf}}=143 \pm 14$ (stat.) \pm 22 (syst.) \pm 3 (lumi.) pb, in agreement with SM expectations.

The lepton+jets final state represents a good compromise between having a large sample and a reasonable purity. In this final state events are required to have one isolated, high transverse momentum electron or muon, large missing transverse energy, and hadronic jets, at least one of which must be consistent with having originated from a $b$ quark. The $t \bar{t}$ cross section is measured using a profile likelihood fit to the number of reconstructed jets, the number of b-tagged jets, and the secondary vertex mass distribution [4]. The measured cross section for an assumed top quark mass of $172.5 \mathrm{GeV}$ is $\sigma_{\mathrm{t} \overline{\mathrm{t}}}=158.1 \pm 2.1$ (stat.) \pm 10.2 (syst.) \pm 3.5 (lumi.) $p b$, which is in agreement with the SM predictions based on the full next-to-leading-order (NLO) matrix elements and the resummation of the leading and NLO soft logarithms.

A similar measurement was performed at $\sqrt{s}=8 \mathrm{TeV}$ using data corresponding to an integrated luminosity of up to $2.8 \mathrm{fb}^{-1}$, collected by the CMS detector at the Large Hadron Collider. The measured inclusive $\bar{t} \bar{t}$ cross section is: $\sigma_{\mathrm{t} \overline{\mathrm{t}}}=228.4 \pm 9.0$ (stat. ${ }_{-26.0}^{+29.0}$ (syst.) \pm 10 (lumi.) $p b$, in agreement with QCD predictions up to approximate next-to-next-to-leading order. 

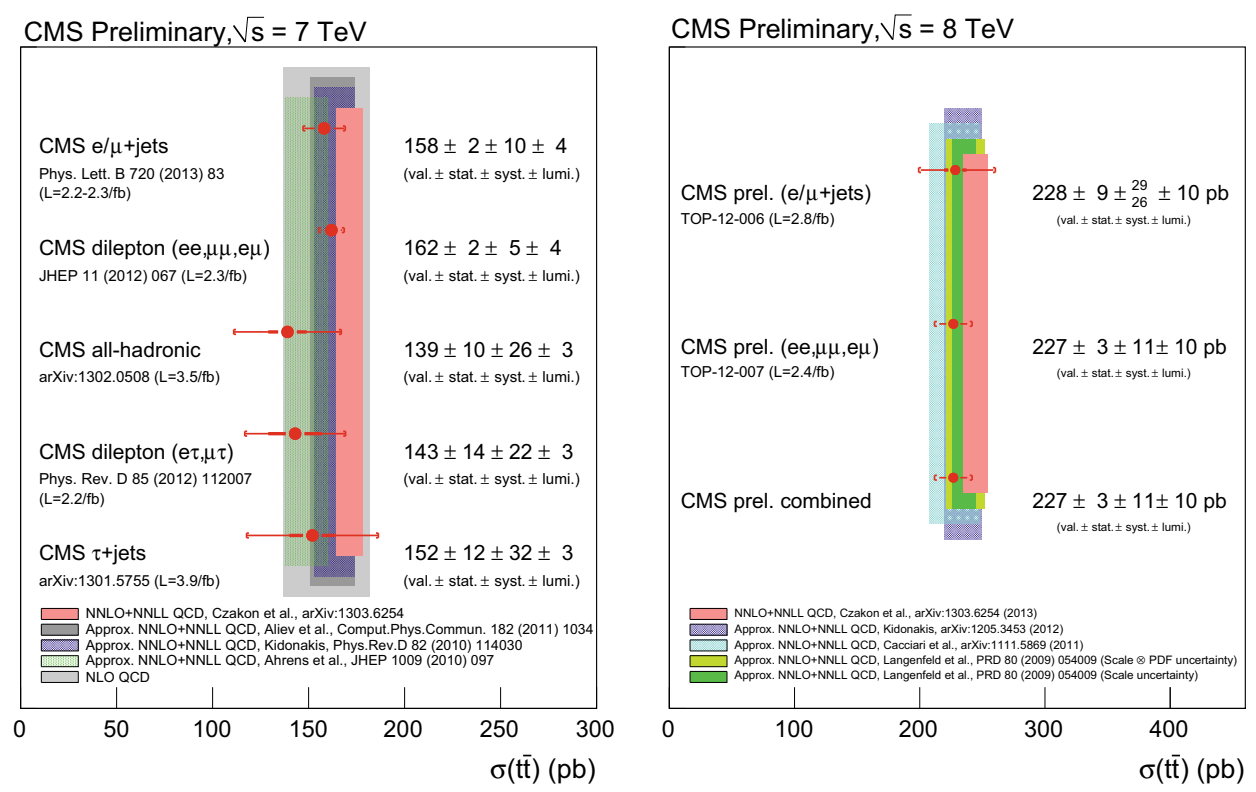

Figure 2. Values of the $\mathrm{tt}^{\mathrm{t}}$ cross section measurement in different final states (left) at $7 \mathrm{TeV}$ and (right) at $8 \mathrm{TeV}$.

Top-quark pairs in the $\tau_{\mathrm{h}}+$ jets final state [5] have been selected in a data sample at $\sqrt{s}=7 \mathrm{TeV}$, corresponding to an integrated luminosity of $3.9 \mathrm{fb}^{-1}$. Events were recorded by a dedicated multijet plus $\tau_{\mathrm{h}}$ trigger, where events are selected with a moderate amount of $E_{\mathrm{T}}^{\mathrm{miss}}$ and four jets, at least one of which is b-tagged. The multijet background is discriminated against using an artificial neural network technique. The result, $\sigma_{\mathrm{tt}}=152 \pm 12$ (stat.) \pm 32 (syst.) $\pm 3 \mathcal{L} \mathrm{pb}$, is consistent with CMS measurements performed in other $\bar{t} \bar{t}$ final states as well as with the SM predicition. The process is the dominant background to a charged Higgs search, where a significant deviation from the SM expectations would indicate the presence of new phenomena.

Assuming a top-quark mass of $172.5 \mathrm{GeV} / \mathrm{c}^{2}$, a first measurement of the t't production cross section in the all-jet channel at $\sqrt{s}=7 \mathrm{TeV}$ yields $\sigma_{\mathrm{t} \overline{\mathrm{t}}}=139 \pm 10$ (stat.) \pm 26 (syst.) $\pm 3 \mathcal{L} \mathrm{pb}$. This result is consistent within $2 \mathrm{SD}$ of an alternative analysis using a neural-network-based selection, and with previous CMS measurements in dilepton and lepton+jets final states, as well as with the predictions of the standard model.

Some of these measurements have been combined at $\sqrt{s}=7 \mathrm{TeV}$ and $\sqrt{s}=8 \mathrm{TeV}$ as can be seen in figure 2 .

Another set of measurements have been performed of normalised differential top-quark production cross section in the $\ell+$ jets (e+jets and $\mu+$ jets) and $\mathrm{e}^{+} \mathrm{e}^{-}, \mu^{+} \mu^{-}$and $\mathrm{e}^{ \pm} \mu^{\mp}$ decay channels. The normalised $\bar{t}$ cross section is measured as a function of the transverse momentum, (pseudo)rapidity, and invariant mass of the final- state leptons and $b$ jets in the visible phase space, and of the top quarks and $\bar{t}$ system in the full phase space. The measurements among the different decay channels are in agreement with each other and with standard model predictions up to approximate next-to-next-toleading- order precision as can be seen in figure 3. The prediction at approximate NNLO precision is found to give a particularly good description of the top-quark transverse momentum. 

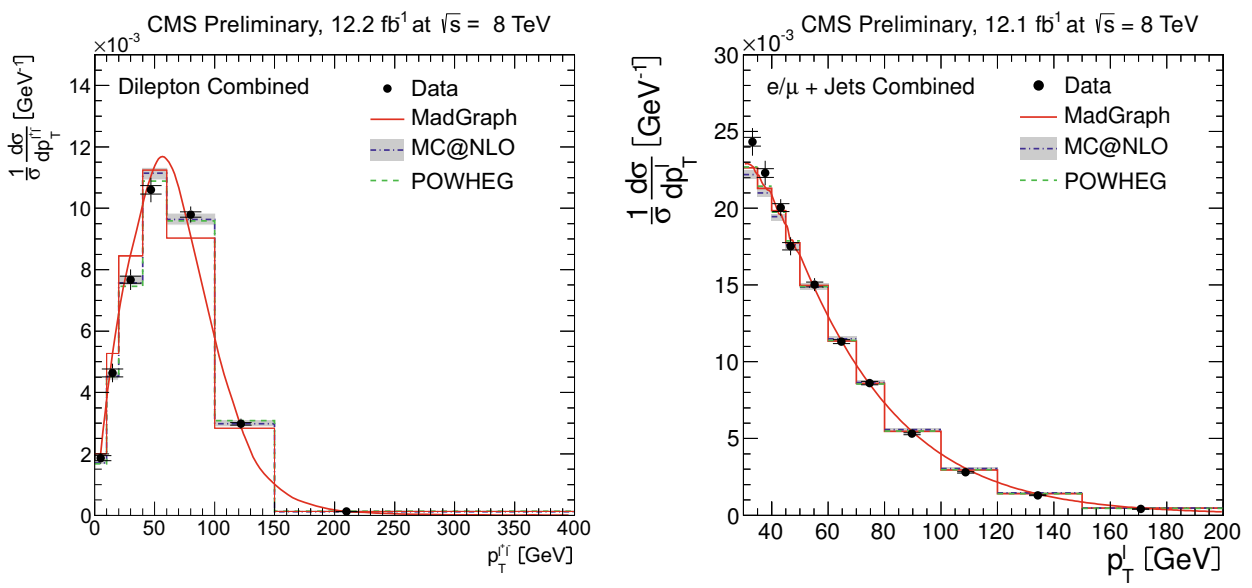

Figure 3. Differential top quark cross section as a function of the dilepton $p_{T}$ in the dilepton channels and the $p_{T}$ of the lepton in the $e / \mu+$ jets final state

\section{Single-top cross section measurements and associated production of top-quark and bosons}

Evidence [7] is presented for the associated production of a single top quark and $\mathrm{W}$ boson in pp collisions at $\sqrt{s}=7 \mathrm{TeV}$ with the CMS experiment at the LHC. The analyzed data corresponds to an integrated luminosity of $4.9 \mathrm{fb}^{-1}$. The measurement is performed using events with two leptons and a jet originated from a $b$ quark. A multivariate analysis based on kinematic properties is utilized to separate the $t \bar{t}$ background from the signal. The observed signal has a significance of $4.0 \mathrm{\sigma}$ and corresponds to a cross section of $16_{-4}^{+5} \mathrm{pb}$, in agreement with the standard model expectation of $15.6 \pm$ $0.4_{-1.2}^{+1.0} \mathrm{pb}$.

The cross section of $t$-channel single-top-quark production [8] has been measured in pp collisions using 2011 data in semileptonic top-quark decay modes with improved precision compared to the previous CMS measurement.Two approaches have been adopted. One approach has been based on a fit of the characteristic pseudorapidity distribution of the light quark recoiling against the single top quark in the $t$-channel with background determination from data. The other has been based on two multivariate discriminators, a Neural Network and Boosted Decision Trees. The multivariate analyses reduce the impact of systematic uncertainties by simultaneously analysing phase space regions with substantial $t$-channel single-top-quark contributions, and regions where they are negligible.The results are all consistent within uncertainties. As a consequence, all three analyses have been combined with the Best Linear Unbiased Estimator method to obtain the final result. The combined measurement of the single-top-quark $t$-channel cross section is $67.2 \pm 6.1 \mathrm{pb}$. It is in agreement with the approximate NNLO standard model prediction of $64.6_{-0.7-1.7}^{+2.1}+1.5 \mathrm{pb}$. Assuming $f_{L_{V}}=1$ and $\left|V_{\mathrm{tb}}\right| \leq 1$, we measure the 95\% confidence level interval $0.92<\left|V_{\mathrm{tb}}\right| \leq 1$.

The production of a single top quark in association with a $\mathrm{W}$ boson is observed for the first time. The analysis uses data collected in pp collisions at $\sqrt{s}=8 \mathrm{TeV}$ by the CMS experiment at the LHC, corresponding to an integrated luminosity of $12.2 \mathrm{fb}^{-1}$. An excess of events above background is found with a significance of $6.1 \sigma$, and a cross section of $23.4_{-5.4}^{+5.5} \mathrm{pb}$ is measured, in agreement with the standard model prediction. 
ICNFP 2013

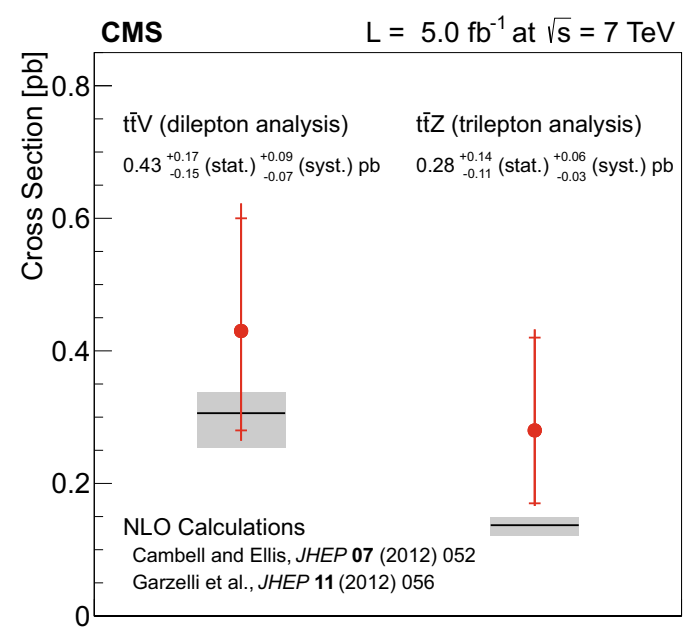

Figure 4. Measurements of the $\mathrm{t} \overline{\mathrm{Z}} \mathrm{Z}$ and $\mathrm{t} \overline{\mathrm{t}} V$ production cross sections, in the same-sign dilepton (left) and trilepton channel (right), respectively. The measurements are compared to the NLO calculations (horizontal black lines) and their uncertainty (grey bands). Internal error bars for the measurements represent the statistical component of the uncertainty.

The first measurement of the cross section of vector boson production associated with a top quarkantiquark pair at $\sqrt{s}=7 \mathrm{TeV}$ has been performed [9] . In the trilepton channel a direct measurement of the $\overline{\mathrm{t} Z \mathrm{Z}}$ cross section $\sigma_{\mathrm{ttZ}}=0.28_{-0.11}^{+0.14}$ (stat.) ${ }_{-0.03}^{+0.06}$ (syst.) pb is obtained, with a significance of 3.3 standard deviations from the background hypothesis. In the dilepton channel a measurement of the $\overline{\mathrm{tt}} V$ process yields $\sigma_{\mathrm{tt} V}=0.43_{-0.15}^{+0.17}$ (stat.) ${ }_{-0.07}^{+0.09}$ (syst.) pb, with a significance of 3.0 standard deviations from the background hypothesis. Both cross section measurements are compatible with the NLO predictions. These results are summarized in Fig. 4.

\section{Top-quark mass measurements}

The top-quark mass $\left(m_{\mathrm{t}}\right)$ is an essential parameter of the standard model. Its measurement also provides an important benchmark for the performance and calibration of the Compact Muon Solenoid (CMS) detector [1]. The top-quark mass has been determined with a high precision at the Fermilab Tevatron to be $m_{\mathrm{t}}=173.18 \pm 0.94 \mathrm{GeV}$ from $\mathrm{t} \overline{\mathrm{t}}$ events in $\mathrm{p} \overline{\mathrm{p}}$ collisions [11]. At the CERN Large Hadron Collider (LHC), the CMS Collaboration has measured the top-quark mass in the dilepton channel where the latest measurement with an integrated luminosity of $5.0 \mathrm{fb}^{-1}$ yields $m_{\mathrm{t}}=172.5 \pm 1.5 \mathrm{GeV}$ [12]. The ATLAS Collaboration has published a measurement in the lepton + jets channel with an integrated luminosity of $1.04 \mathrm{fb}^{-1}$, also using a template method, that gives $m_{\mathrm{t}}=174.5 \pm 2.4 \mathrm{GeV}[13]$.

In the analysis presented in [10], we select events containing top-quark pairs where each top quark decays weakly via $\mathrm{t} \rightarrow \mathrm{bW}$, with one $\mathrm{W}$ boson decaying into a charged lepton and its neutrino, and the other into a quark-antiquark pair. Hence, the final state consists of a lepton, four jets, and an undetected neutrino. The analysis employs a kinematic fit of the decay products to a $\mathrm{t}^{\bar{t}}$ hypothesis and two-dimensional (2D) likelihood functions for each event to estimate simultaneously both the 


\section{EPJ Web of Conferences}

top-quark mass and the jet energy scale (JES). The invariant mass of the two jets associated with the hadronic decay serves as an additional observable in the likelihood functions to estimate the JES directly exploiting the precise knowledge of the W-boson mass from previous measurements [14].

The complete kinematic properties of each event are reconstructed through a constrained fit to a tit hypothesis. For each selected event, a likelihood is calculated as a function of assumed values of the top-quark mass and the jet en ergy scale, taking into account all possible jet assignments. From a data sample corresponding to an integrated luminosity of $5.0 \mathrm{fb}^{-1}, 5174$ candidate events are selected and the top-quark mass is measured to be $m_{\mathrm{t}}=173.49 \pm 0.43$ (stat. $+\mathrm{JES}$ ) \pm 0.98 (syst.) GeV. This result is consistent with the Tevatron average [11], and constitutes the most precise single measurement to date of the mass of the top quark, a combination of the different measurements performed in CMS are compared to World Average (Tevatron) in figure 6:

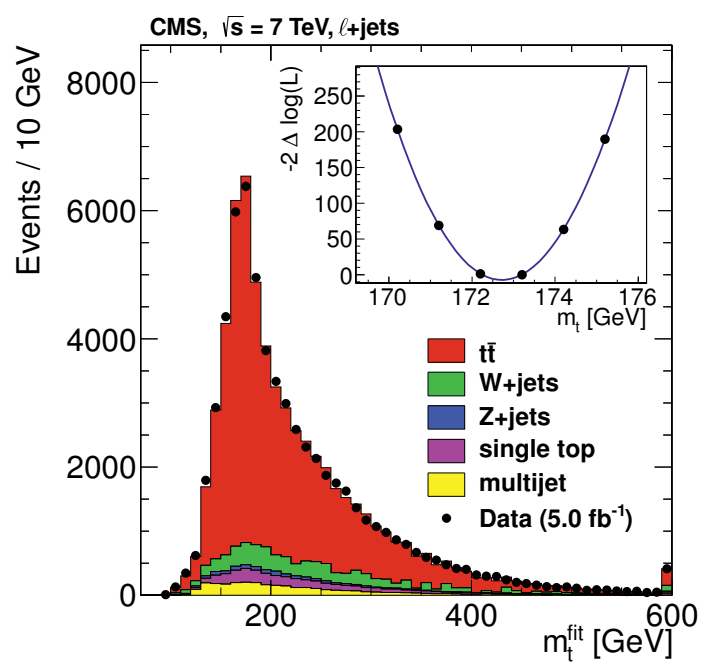

Figure 5. Distribution of the reconstructed top-quark mass after the kinematic fit, for the permutation with the lowest $\chi^{2}$, in the cross-check analysis. The distributions are normalized to the number of events observed in data. The top-quark mass assumed in the simulation is $172.5 \mathrm{GeV}$. The rightmost bin also contains the overflow. The inset shows the cross-check likelihood measured for the $\ell+$ jets final state.

\section{Summary}

The CMS detector has developed a full program of measurements related with the top-quark at 7 and $8 \mathrm{TeV}$ using the luminosity collected at both energies during the years 2011 and 2012. Top-quark physics is a crucial part of the current research program in HEP, key to QCD, EWK and searches for new physics. It constitutes an ideal probe to look for new physics beyond the SM with precision measurements or with direct searches for new signals. The results presented here summarises what the CMS experiment has measured in the two last years with proton proton collisions at the LHC collider, allowing to enter in a precision regime in which cross sections have been measured with uncertainties below $5 \%$ and the top quark mass has been measured with precision below $1 \mathrm{GeV}$. 


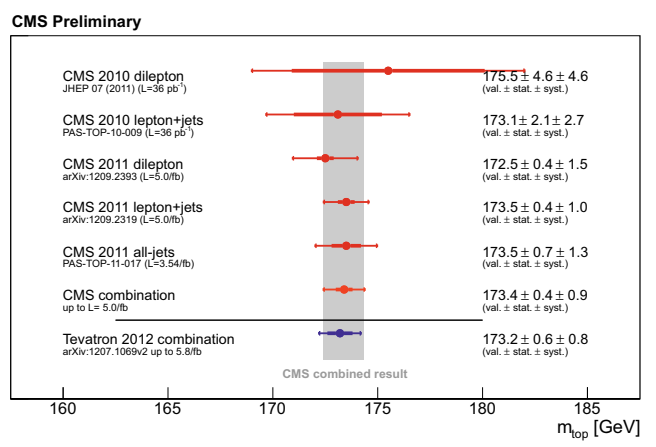

Figure 6. Values of different top-quark mass measurements performed in CMS compared with the world average mass dominated by Tevatron measurements.

\section{References}

[1] Chatrchyan, S. and others, JINST 3, S08004 (2008)

[2] Chatrchyan, S. and others, JHEP 11, 067 (2012)

[3] Chatrchyan, S. and others, Phys. Rev. D 85, 112007 (2012)

[4] Chatrchyan, S. and others, Phys. Lett. B 83, 720 (2013)

[5] Chatrchyan, S. and others, Eur. Phys. J. C 73, 2386 (2013)

[6] Chatrchyan, S. and others, JHEP 05, 065 (2013)

[7] Chatrchyan, S. and others, Phys. Rev. Lett. 110, 022003 (2013)

[8] Chatrchyan, S. and others, JHEP 12, 035 (2012)

[9] Chatrchyan, S. and others, Phys. Rev. Lett. 110, 172002 (2013)

[10] Chatrchyan, S. and others, JHEP 12, 105 (2012)

[11] CDF and DØ Collaborations, Phys. Rev. D 86, 092003 (2012)

[12] Chatrchyan, S. and others, Eur. Phys. J. C 72, 2202 (2012)

[13] Aad, G. and others, Eur. Phys. J. C 72, 2046 (2012)

[14] Beringer, J. and others, Phys. Rev. D 86, 010001 (2012) 